Communication

\title{
Heteronuclear dipolar recoupling in multiple-spin system under fast magic-angle spinning
}

\author{
Fang-Chieh Chou, Shing-Jong Huang, Jerry C.C. Chan* \\ Department of Chemistry, National Taiwan University, No. 1, Section 4, Roosevelt Road, Taipei, Taiwan
}

\section{A R T I C L E I N F O}

\section{Article history:}

Received 18 September 2008

Revised 21 November 2008

Available online 3 December 2008

\section{Keywords:}

C-REDOR

Rotary-REDOR

Heteronuclear dipolar recoupling

MAS

\begin{abstract}
A B S T R A C T
We show that the C-REDOR sequence can be modified according to the framework of rotary-REDOR to realize heteronuclear recoupling in multiple-spin systems. Because of the inherent favorable properties of C-REDOR with respect to rf inhomogeneity, our approach is anticipated to find useful applications in biological research. Furthermore, active rotor synchronization of the windowless C-REDOR is implemented by an ad hoc shortening of the pulse elements. Numerical simulations and experiments measured for $\left[\mathrm{U}-{ }^{13} \mathrm{C},{ }^{15} \mathrm{~N}\right]$-L-alanine are used to validate our approach.
\end{abstract}

(c) 2008 Elsevier Inc. All rights reserved.

\section{Introduction}

Heteronuclear distance measurements under the condition of magic-angle spinning (MAS) have many important applications in biological research [1-3]. The most widely applied pulse sequence developed to date for heteronuclear dipole-dipole recoupling is the rotational double resonance (REDOR) technique [4]. The major disadvantage of REDOR is that homonuclear dipole-dipole interaction in multiple-spin systems might interfere with the heteronuclear dipolar dephasing in the regime of fast MAS spinning $(\geqslant 20 \mathrm{kHz}$ ). Thanks to the novel pulse symmetries developed by Levitt and coworkers [5-7], a series of methods based on the $C$ and $R$ symmetries were developed to extend the concept of heteronuclear dipolar recoupling to multiple-spin systems under fast MAS condition [8-14]. Recently, it has been shown that the rotary resonance [15] can be combined with REDOR for distance measurement under fast MAS condition [16]. The major advantage of rotary-REDOR is that the pulse sequence allows for measurements involving quadrupole nuclei. The rotary-REDOR $(n=2)$ technique has further been applied for heteronuclear recoupling in multiple-spin systems [17]. On the other hand, it has been documented that rotary resonance is quite sensitive to experimental imperfections $[15,18]$. In this work, we show that the C-REDOR sequence $[8,9]$ can be modified according to the framework of rotary-REDOR to realize heteronuclear recoupling in multiple-spin systems. Because of the inherent favorable properties of C-REDOR with respect to rf inhomogeneity, the

\footnotetext{
* Corresponding author. Fax: +88622363 6359.

E-mail address: chanjcc@ntu.edu.tw (J.C.C. Chan).
}

pulse sequence shown in Fig. 1 is anticipated to find useful applications in biological research. However, active rotor synchronization, which is highly desirable for quantitative analysis of dephasing curves, is not easy to implement for windowless pulse sequences like C-REDOR. Here, we suggest to add the triggers of the tachometer signals by an ad hoc shortening of the pulse elements in windowless pulse sequences. In the following sections, numerical simulations and experiments measured for $\left[\mathrm{U}-{ }^{13} \mathrm{C},{ }^{15} \mathrm{~N}\right]$-L-alanine are used to validate our approach.

\section{Experimental methods}

All NMR experiments were carried out at ${ }^{13} \mathrm{C}$ and ${ }^{1} \mathrm{H}$ frequencies of 75.5 and $300.1 \mathrm{MHz}$, respectively, on a Bruker DSX300 NMR spectrometer equipped with a commercial $2.5 \mathrm{~mm}$ triple-resonance probe. The magic-angle spinning (MAS) frequency was set to $25 \mathrm{kHz}$ and its variation was limited to $\pm 2 \mathrm{~Hz}$ using a commercial pneumatic control unit (Bruker, MAS II). The sample was confined in the middle one-half of the rotor volume using Teflon spacers. Typically, during the cross polarization contact time $(1.5 \mathrm{~ms})$, the ${ }^{1} \mathrm{H}$ nutation frequency was set to $50 \mathrm{kHz}$ and that of ${ }^{13} \mathrm{C}$ was ramped through the Hartmann-Hahn matching condition $[19,20]$. Continuous-wave and XiX [21] proton decouplings of $120 \mathrm{kHz}$ were applied during recoupling periods and the $t_{2}$ acquisition, respectively. During the C-REDOR recoupling period, the ${ }^{13} \mathrm{C}$ nutation frequency was set to $52.6 \mathrm{kHz}$. The ${ }^{13} \mathrm{C}$ and ${ }^{15} \mathrm{~N} \pi$ pulses were set to 6.8 and $16 \mu$ s, respectively. The tachometer signals for active rotor synchronization were filtered with a homebuilt phase-locked loop circuit. 

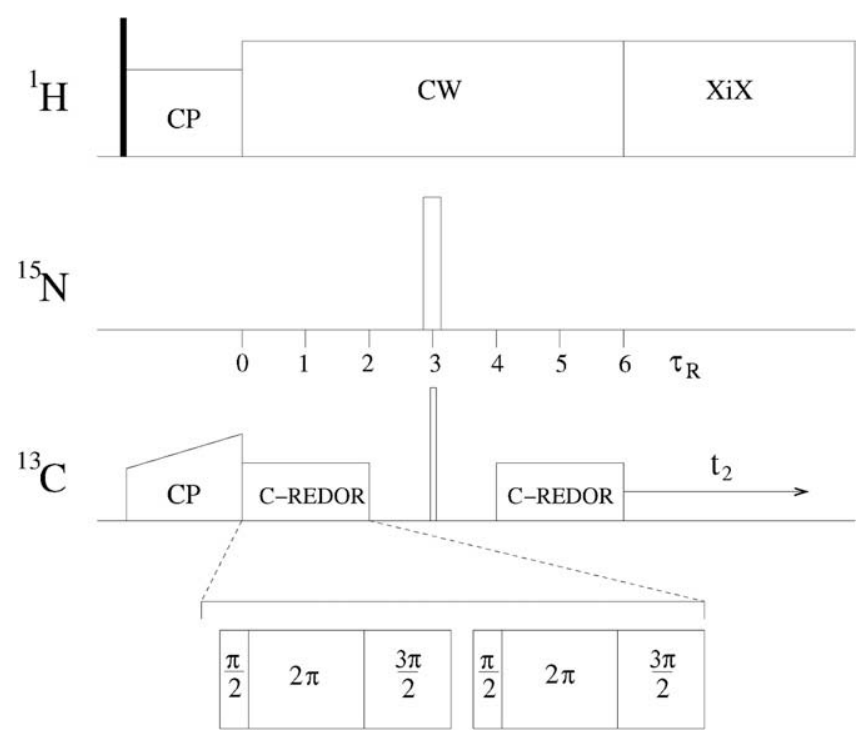

Fig. 1. Pulse sequence used for heteronuclear dipolar recoupling in multiple-spin systems. The rectangle in black denotes a $\pi / 2$ pulse. The open rectangles near the middle of the ${ }^{13} \mathrm{C}$ and ${ }^{15} \mathrm{~N}$ channels represent $\pi$ pulses. The C-REDOR sequence spanning two rotor periods contains two POST-blocks. A delay of $2 \mu \mathrm{s}$ was inserted between two adjacent blocks for active rotor synchronization.

Numerical simulations were carried out using the package SIMPSON (version 1.1.0) [22]. The maximum time step over which the Hamiltonian is approximated to be time-independent was set to 1.0. Typically, a powder averaging scheme containing 320 REPULSION angles $(\alpha$ and $\beta$ ) [23] and $18 \gamma$ angles was chosen. Relaxation effects were ignored. The geometry and NMR parameters of the spin systems were set with reference to L-alanine [24,25].

\section{Results and discussion}

\subsection{Active rotor synchronization in C-REDOR}

In general, $\mathrm{CN}_{n}^{v}$ pulse sequences comprise $N$ repetitions of a subcycle $C$, spanning a total of $n$ rotor periods $\left(n \tau_{R}\right)$ [7-9]. $C$ is an rf sequence of length $\tau_{\mathrm{C}}=n \tau_{R} / \mathrm{N}$, comprising pulses that the net rotation in each subcycle is an integer multiple of $2 \pi$. An overall $\mathrm{rf}$ phase shift $2 \pi q v / N$ is applied to the $q$ th repetition of $C$, where $q$ is an integer running from 0 to $N-1$. In the C-REDOR, the POST-composite pulse [26] is incorporated into each subcycle of the $C$ sequence with symmetry $\mathrm{C} n_{n}^{1}$. The $\mathrm{C} 2{ }_{2}^{1}$ symmetry is chosen to reduce the overall cycle time to $2 \tau_{\mathrm{R}}$. For windowless sequences like C-REDOR, active rotor synchronization may be implemented by shortening the rf pulses. We find that a delay of $2 \mu$ s inserted between two adjacent POST-blocks is long enough to provide a stable timing for the pulse train of C-REDOR in our spectrometer. To restrict the time span of each C-REDOR cycle to $2 \tau_{R}$, we may either slightly increase the rf nutation frequency to keep the flip angles intact, or shorten the flip angle of each pulse. In either case, the error involved in the nutation frequency or the flip angle is about $5 \%$ at a spinning frequency of $25 \mathrm{kHz}$.

\subsection{Numerical simulations}

We carried out the simulation of a ${ }^{13} \mathrm{C}-{ }^{15} \mathrm{~N}$ system on the basis of the C-REDOR sequence shown in Fig. 1. The spinning frequency was set to $25 \mathrm{kHz}$. The ${ }^{13} \mathrm{C}$ nutation frequency during the C-REDOR recoupling period was set to $52.6 \mathrm{kHz}$, which is slightly higher than the nominal value of $50 \mathrm{kHz}$ according to the symmetry of POST$\mathrm{C}_{2}{ }_{2}^{1}$. For comparison, we also simulate the dephasing curve with the C-REDOR recoupling field set equal to $50 \mathrm{kHz}$. Specifically, the three pulses in a POST-block, viz. $\pi / 2,2 \pi$, and $3 \pi / 2$, are shortened by $0.25,1$, and $0.75 \mu$ s, respectively. With reference to Fig. 2 , the overall recoupling efficiency of C-REDOR is quite comparable to that of rotary-REDOR $(n=2)$ and the performance is not significantly affected by the insertion of the short delays for active rotor synchronization. Fig. 3 shows the modulation of the recoupling efficiency of the delay-inserted C-REDOR with respect to rf inhomogeneity. It is interesting to find that our approach is more forgiving to the cases in which the rf fields are smaller than the nominal value, indicating that a severe restriction of the sample volume is not necessary. This favorable feature is originated from the pulse symmetry of the C-REDOR [9]. Although homonuclear dipole-dipole interaction is suppressed in the original implementation of C-REDOR $[8,9,27]$, the simulations shown in Fig. 4a reveal

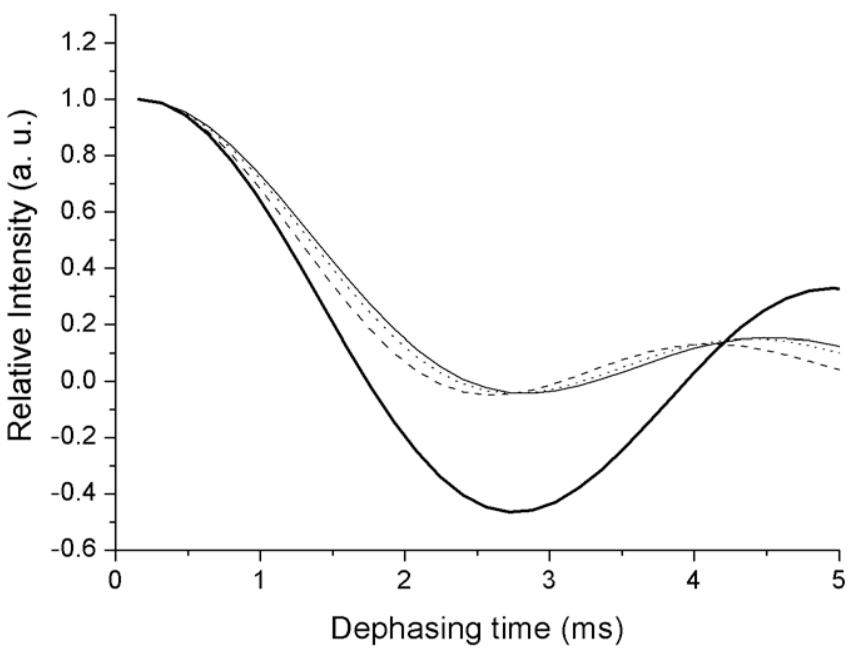

Fig. 2. Dephasing curves calculated for a ${ }^{13} \mathrm{C}_{\alpha}-{ }^{15} \mathrm{~N}$ spin system. The dipolar coupling constant was set to $933.56 \mathrm{~Hz}$. All the simulations were calculated at a spinning frequency of $25 \mathrm{kHz}$ on the basis of the pulse sequence shown in Fig. 1. The ${ }^{13} \mathrm{C}$ and ${ }^{15} \mathrm{~N} \pi$ pulses were set to 6.8 and $16 \mu$ s, respectively. The dashed and the dotted lines correspond to the cases in which the ${ }^{13} \mathrm{C}$ rf nutation frequency was set to 50 and $52.6 \mathrm{kHz}$, respectively. The thin solid line corresponds to the original windowless C-REDOR sequence. The thick solid line denotes the dephasing calculated for the rotary-REDOR $(n=2)$.

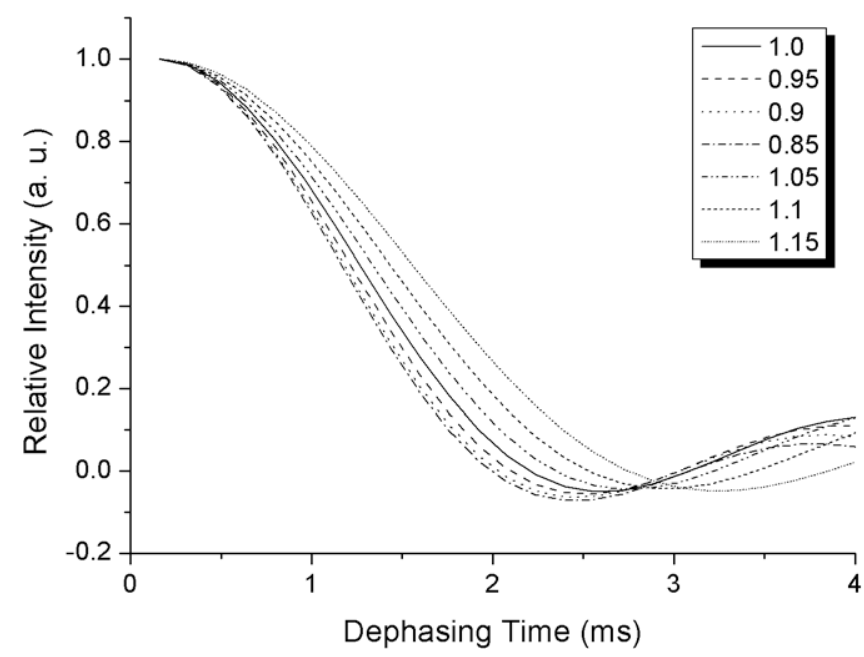

Fig. 3. Effects of rf inhomogeneity on the C-REDOR dephasing. The rf field strength is set deviated from the nominal value by a factor varied from 0.85 to 1.15 . Other simulation parameters are referred to the caption of Fig. 2. 

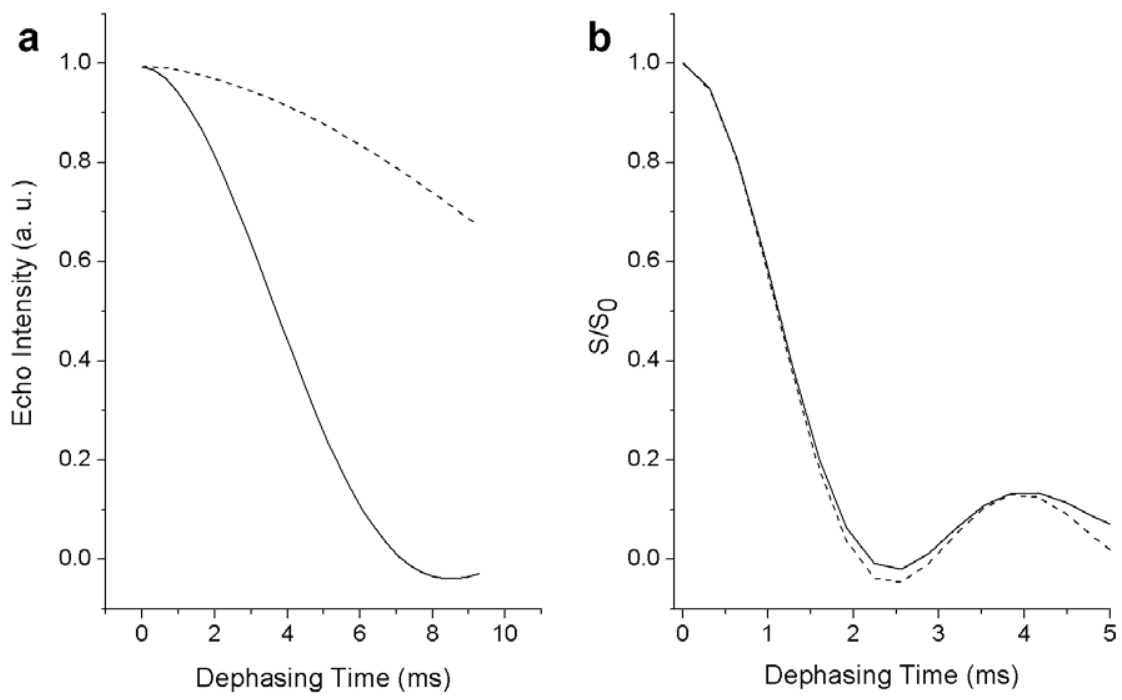

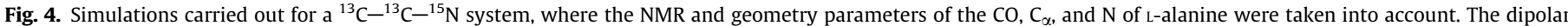

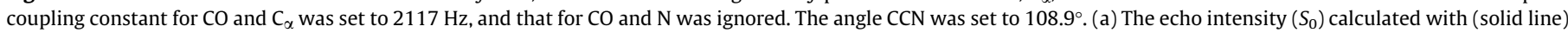

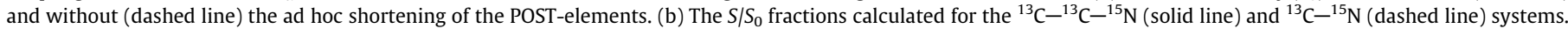
Other simulation parameters are referred to the caption of Fig. 2.

that the insertion of short delays will cause an additional attenuation of the reference signal $\left(S_{0}\right)$. In other words, the major disadvantage of our ad hoc procedure is that the scaling factor of homonuclear dipole-dipole interaction will become non-zero, causing a faster signal decay of the reference signal. Nevertheless, the calculated C-REDOR fractions $\left(S / S_{0}\right)$ for the ${ }^{13} \mathrm{C}-{ }^{13} \mathrm{C}-{ }^{15} \mathrm{~N}$ system are very similar to those calculated for ${ }^{13} \mathrm{C}-{ }^{15} \mathrm{~N}$ for short dephasing times (Fig. 4b). This finding makes it possible to analyze the experimental data of uniformly ${ }^{13} \mathrm{C}$ labeled systems by the model of an isolated ${ }^{13} \mathrm{C}-{ }^{15} \mathrm{~N}$ spin pair. Because we have added a very short delay at the beginning of each $C$ subcycle, it would be interesting to investigate whether the performance of our approach would heavily dependent on the stability of the spin rate. Fig. 5 shows the calculated REDOR fractions for a ${ }^{13} \mathrm{C}-{ }^{13} \mathrm{C}-{ }^{15} \mathrm{~N}$ system at different spinning frequencies. It is satisfying to find that the dephasing is not very sensitive to the fluctuation of spin rate up to $\pm 100 \mathrm{~Hz}$ at a spinning frequency of $25 \mathrm{kHz}$.

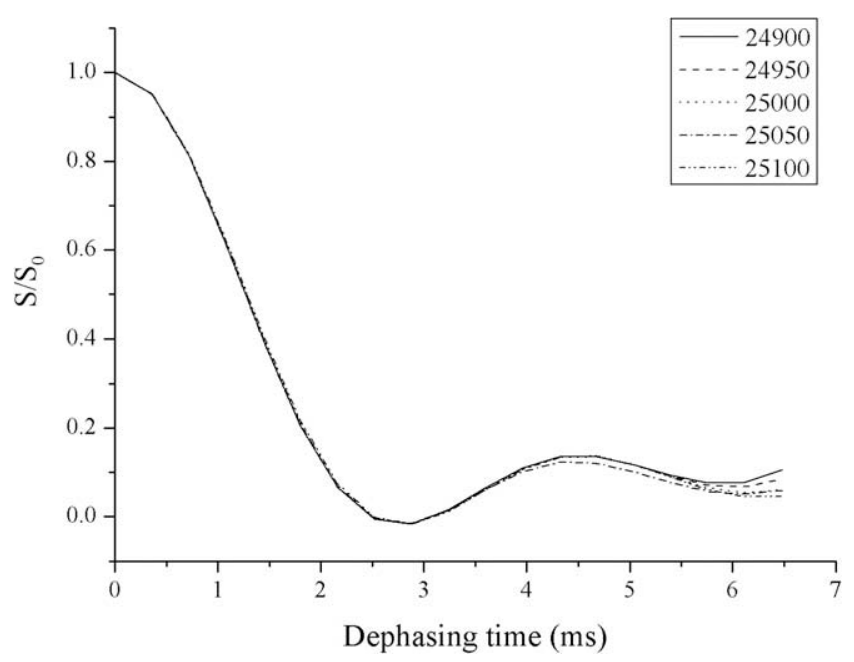

Fig. 5. Simulations carried out for a ${ }^{13} \mathrm{C}-{ }^{13} \mathrm{C}-{ }^{15} \mathrm{~N}$ system at different spinning frequencies, varied from 25,100 to $24,900 \mathrm{~Hz}$. Other simulation parameters are referred to the caption of Fig. 4.

\subsection{Experimental verification}

Fig. 6 shows the experimental data we obtained for the ${ }^{13} \mathrm{C}_{\alpha}$ of $\left[\mathrm{U}-{ }^{13} \mathrm{C},{ }^{15} \mathrm{~N}\right]-\mathrm{L}-$ alanine. In comparison with the Hahn echo signal intensities, our reference signals are indeed attenuated considerably (data not shown). Nevertheless, the experimental C-REDOR fractions obtained are in good agreement with the simulations carried out on a ${ }^{13} \mathrm{C}-{ }^{15} \mathrm{~N}$ spin-pair system. Although the spin-rate stability provided by our pneumatic control unit is reasonably high, empirically we find that it is necessary to implement active rotor synchronization to reduce signal fluctuation. Our approach to provide active rotor synchronization for windowless pulse sequences is rather straightforward to implement. In summary, the C-REDOR implementation discussed in this work is rather robust and can be applied to uniformly ${ }^{13} \mathrm{C}$ labeled systems. Extension to other ${ }^{13} \mathrm{C}-{ }^{15} \mathrm{~N}$ systems with longer spin-spin distances or larger ${ }^{13} \mathrm{C}$ chemical shift anisotropy is currently under investigation. We

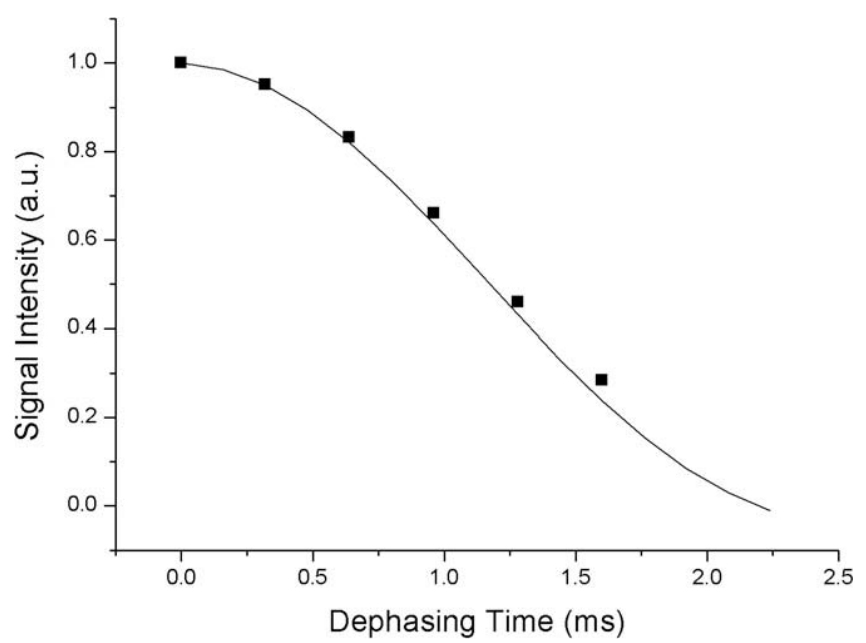

Fig. 6. Experimental (squares) and simulation data (solid line) obtained for the ${ }^{13} \mathrm{C}_{\alpha}$ of $\left[\mathrm{U}-{ }^{13} \mathrm{C},{ }^{15} \mathrm{~N}\right]$-L-alanine at a spinning frequency of $25 \mathrm{kHz}$. The simulation was carried for the ${ }^{13} \mathrm{C}_{\alpha}-{ }^{15} \mathrm{~N}$ system of L-alanine, where all the rf field strengths and durations were matched to the experimental conditions. 
expect that our method can also be applied for quadrupole systems as illustrated in rotary-REDOR $[16,17,28,29]$.

\section{Acknowledgments}

This work was supported by grants from the National Science Council. F.C.C. acknowledges an undergraduate stipend from NSC.

\section{References}

[1] M. Baldus, Correlation experiments for assignment and structure elucidation of immobilized polypeptides under magic angle spinning, Prog. Nucl. Magn. Reson. Spectrosc. 41 (2002) 1.

[2] R. Tycko, Applications of solid state NMR to the structural characterization of amyloid fibrils: methods and results, Prog. Nucl. Magn. Reson. Spectrosc. 42 (2003) 53.

[3] R. Tycko, Progress towards a molecular-level structural understanding of amyloid fibrils, Curr. Opin. Struct. Biol. 14 (2004) 96.

[4] T. Gullion, J. Schaefer, Rotational-echo double-resonance NMR, J. Magn. Reson. 81 (1989) 196.

[5] Y.K. Lee, N.D. Kurur, M. Helmle, O.G. Johannessen, N.C. Nielsen, M.H. Levitt, Efficient dipolar recoupling in the NMR of rotating solids-a sevenfold symmetrical radiofrequency pulse sequence, Chem. Phys. Lett. 242 (1995) 304

[6] M. Carravetta, M. Eden, X. Zhao, A. Brinkmann, M.H. Levitt, Symmetry principles for the design of radiofrequency pulse sequences in the nuclear magnetic resonance of rotating solids, Chem. Phys. Lett. 321 (2000) 205.

[7] M.H. Levitt, Symmetry-based pulse sequence in magic-angle spinning solidstate NMR, in: D.M. Grant, R.K. Harris (Eds.), Encyclopedia in Nuclear Magnetic Resonance, Wiley, Chichester, 2002, pp. 165-196.

[8] J.C.C. Chan, C-REDOR: rotational echo double resonance under very fast magicangle spinning, Chem. Phys. Lett. 335 (2001) 289.

[9] J.C.C. Chan, H. Eckert, C-rotational echo double resonance: heteronuclear dipolar recoupling with homonuclear dipolar decoupling, J. Chem. Phys. 115 (2001) 6095.

[10] X. Zhao, M. Eden, M.H. Levitt, Recoupling of heteronuclear dipolar interactions in solid-state NMR using symmetry-based pulse sequences, Chem. Phys. Lett. 342 (2001) 353.

[11] X. Zhao, W. Hoffbauer, J. Schmedt auf der Günne, M.H. Levitt, Heteronuclear polarization transfer by symmetry-based recoupling sequences in solid-state NMR, Solid State Nucl. Magn. Reson. 26 (2004) 57.

[12] A. Brinkmann, A.P.M. Kentgens, Proton-selective O-17-H-1 distance measurements in fast magic-angle-spinning solid-state NMR spectroscopy for the determination of hydrogen bond lengths, J. Am. Chem. Soc. 128 (2006) 14758.
[13] A. Brinkmann, A.P.M. Kentgens, Sensitivity enhancement and heteronuclear distance measurements in biological 0-17 solid-state NMR, J. Phys. Chem. B 110 (2006) 16089.

[14] D.H. Brouwer, J.A. Ripmeester, Symmetry-based recoupling of proton chemical shift anisotropies in ultrahigh-field solid-state NMR, J. Magn. Reson. 185 (2007) 173.

[15] M.H. Levitt, T.G. Oas, R.G. Griffin, Rotary resonance recoupling in heteronuclear spin pair systems, Isr. J. Chem. 28 (1988) 271

[16] Z.H. Gan, Rotary resonance echo double resonance for measuring heteronuclear dipolar coupling under MAS, J. Magn. Reson. 183 (2006) 235.

[17] Z.H. Gan, Proton-detected N-14 MAS NMR using homonuclear decoupled rotary resonance, Chem. Phys. Lett. 435 (2007) 163.

[18] T.G. Oas, R.G. Griffin, M.H. Levitt, Rotary resonance recoupling of dipolar interactions in solid-state nuclear magnetic resonance spectroscopy, J. Chem. Phys. 89 (1988) 692

[19] S. Hediger, B.H. Meier, N.D. Kurur, G. Bodenhausen, R.R. Ernst, NMR crosspolarization by adiabatic passage through the Hartmann-Hahn condition (Aphh), Chem. Phys. Lett. 223 (1994) 283.

[20] S. Hediger, B.H. Meier, R.R. Ernst, Adiabatic passage Hartmann-Hahn crosspolarization in NMR under magic-angle sample-spinning, Chem. Phys. Lett. 240 (1995) 449.

[21] A. Detken, E.H. Hardy, M. Ernst, B.H. Meier, Simple and efficient decoupling in magic-angle spinning solid-state NMR: the XiX scheme, Chem. Phys. Lett. 356 (2002) 298.

[22] M. Bak, J.T. Rasmussen, N.C. Nielsen, SIMPSON: a general simulation program for solid-state NMR spectroscopy, J. Magn. Reson. 147 (2000) 296.

[23] M. Bak, N.C. Nielsen, REPULSION, a novel approach to efficient powder averaging in solid-state NMR, J. Magn. Reson. 125 (1997) 132.

[24] M.S. Lehmann, T.F. Koetzle, W.C. Hamilton, Precision neutron diffraction structure determination of protein and nucleic acid components. I. The crystal and molecular structure of the amino acid L-alanine, J. Am. Chem. Soc. 94 (1972) 2657.

[25] A. Naito, S. Ganapathy, K. Akasaka, C.A. McDowell, Chemical shielding tensor and C-13-N-14 dipolar splitting in single-crystals of L-alanine, J. Chem. Phys. 74 (1981) 3190.

[26] M. Hohwy, H.J. Jakobsen, M. Eden, M.H. Levitt, N.C. Nielsen, Broadband dipolar recoupling in the nuclear magnetic resonance of rotating solids: a compensated C7 pulse sequence, J. Chem. Phys. 108 (1998) 2686.

[27] J.C.C. Chan, R. Tycko, Recoupling of chemical shift anisotropies in solid-state NMR under high-speed magic-angle spinning and in uniformly C-13-labeled systems, J. Chem. Phys. 118 (2003) 8378.

[28] Z.H. Gan, Measuring nitrogen quadrupolar coupling with C-13 detected wide-line N-14 NMR under magic-angle spinning, Chem. Commun. (2008) 868.

[29] Z.H. Gan, C-13/N-14 heteronuclear multiple-quantum correlation with rotary resonance and REDOR dipolar recoupling. J. Magn. Reson. 184 (2007) 39. 